\title{
Savoirs et apprentissages fondamentaux à
} Singapour

Knowledge and basic learning in Singapore

Conocimiento y aprendizaje fundamentales en Singapur

Nicole Green, Jen Yi Li et Pei Wen Tzuo

Traducteur : Robert Elbaz

\section{OpenEdition}

\section{Journals}

Édition électronique

URL : http://journals.openedition.org/ries/200

DOI : $10.4000 /$ ries.200

ISSN : 2261-4265

Éditeur

Centre international d'études pédagogiques

\section{Édition imprimée}

Date de publication : 1 décembre 2006

Pagination : 33-45

ISSN : 1254-4590

\section{Référence électronique}

Nicole Green, Jen Yi Li et Pei Wen Tzuo, «Savoirs et apprentissages fondamentaux à Singapour », Revue internationale d'éducation de Sèvres [En ligne], 43 | décembre 2006, mis en ligne le 22 juin 2011 , consulté le 21 avril 2019. URL : http://journals.openedition.org/ries/200 ; DOI : 10.4000/ries.200 


\section{Savoirs \\ et apprentissages fondamentaux à Singapour*}

\section{Nicole Green \\ Jen Yi Li \\ Pei Wen Tzuo**}

Le système éducatif de Singapour a été créé sous l'égide fondatrice du Premier ministre Lee Kuan Yew, qui a reconnu que l'investissement pédagogique était la source essentielle de la richesse et de la prospérité du pays. Le premier gouvernement indépendant de ce pays-île était centralisé, standardisé et hiérarchisé. Il se concentrait sur une éducation orientée vers la survie. En 1979 a commencé une restructuration majeure du système éducatif de Singapour pour en faire un système donnant la priorité à l'efficacité, face aux défis croissants de l'émergence d'économies à faibles coûts de production en Asie du Sud-Est. Ce système éducatif «efficace» a encouragé le développement d'une économie fondée sur les savoirs (Mukhopadhaya, 2003) et mis l'accent sur l'apprentissage par cœur, sur le suivi et l'orientation des élèves, ainsi que sur leur capacité à obéir et à se conformer (Boshier, 1994).

Plus récemment, l'ex-Premier ministre Goh Chok Tong (1997) a fourni le cadre pour nombre des réformes pédagogiques qui ont été mises en place ces dix dernières années, suivant la vision dite «Des écoles qui pensent, une nation qui apprend». Ces réformes se sont donné pour but d'instituer un système éducatif fondé sur les capacités. Elles ont été soutenues et améliorées ultérieurement par le Premier ministre suivant, Lee Hsien Loong, depuis 2004 (Ho, 2006). C'est à cette époque que le ministère de l'éducation de Singapour a défini les grandes lignes des «finalités attendues de l'enseignement», en spécifiant les objectifs premiers de toute éducation : promouvoir la cohésion sociale d'individus d'origines socioculturelles différentes et encourager une identité nationale commune pour les préparer à leur rôle de citoyens adultes.

La description par Tyler (1949) de l'approche retenue pour le programme pédagogique indique que celui-ci décide avant tout des objectifs pédagogiques, puis crée les activités d'apprentissage et l'évaluation pour atteindre ces objectifs. Cette description reflète le paysage pédagogique de Singapour. Le programme national pourrait être décrit comme normatif puisqu'il indique ce qui devrait

\footnotetext{
* Article traduit par Robert Elbaz.

** Les auteurs sont présentés par ordre alphabétique.
} 
être enseigné et évalué, mais aussi ce que l'on valorise. Le système éducatif promeut la sélection et la ventilation des individus au sein de la structure professionnelle de Singapour, servant ainsi à différencier les individus et à jouer une fonction de Cerbère de la société.

\section{LE PROGRAMME NATIONAL}

\section{Les objectifs éducatifs}

Dans la plupart des pays, les buts de l'éducation peuvent généralement être illustrés par l'expression «bon citoyen»(Klein, 1996). Partageant de nombreuses valeurs culturelles avec d'autres pays d'Asie comme la Chine, le Japon et la Corée du Sud, Singapour associe la notion de «bon citoyen» à des individus pleinement responsables de leurs devoirs dans le contexte d'une société collective et qui accordent de la valeur à cette vie collective par leur coopération individuelle (ministère de l'éducation, 2006). Considérant que l'éducation reflète les objectifs de la société, le ministère indique que non content d'inciter chaque élève à apprendre et à se développer jusqu'à atteindre un haut degré de réussite scolaire, l'accent est également mis sur la capacité de chacun à s'aligner sur la famille, la communauté et le pays. En retour, les écoles de Singapour doivent concevoir un programme qui tienne compte des besoins et des intérêts de chacun, tout en prenant en compte les valeurs fondamentales de la société comme entité cohérente, tout en respectant la politique éducative et les normes culturelles d'éducation (telles que la valeur accordée à la réussite scolaire).

Les objectifs pédagogiques principaux de Singapour ont été définis autour du maintien de l'ordre social, répondant aux valeurs fondamentales de responsabilité, de coopération et de cohésion sociale. Plus récemment, ils mettent aussi l'accent sur la promotion des intérêts, des capacités, des talents divers des élèves, ainsi que sur leur créativité. Durant les dix dernières années, cessant de se concentrer exclusivement sur l'apprentissage des matières de base, les réformes pédagogiques ont cherché à favoriser diverses capacités et à promouvoir la créativité des élèves. Cet effort a été encouragé par une prise de conscience croissante que la façon traditionnelle d'enseigner (c'est-à-dire par instructions directes) étouffait la créativité des élèves et excluait toute une variété de façons d'apprendre et de savoir. Ces efforts nouveaux peuvent être perçus dans les réformes récentes, telles que le slogan «enseigner moins, apprendre plus", et l'initiative $\mathrm{SEED}^{1}$ (Students' Engagement for Effective Development - Engagement des élèves pour un développement efficace). Toutefois, les éducateurs font face à des difficultés lorsqu'il s'agit de modifier leur approche de l'enseignement et de l'apprentissage, et de passer de l'apprentissage traditionnel passif fondé sur les exercices répétitifs et le par cœur à une pédagogie différente qui réponde aux exigences du nouveau programme.

1. NdT : jeu de mots intraduisible : le mot anglais «seed» signifie «graine, semence». 


\section{L'encouragement des pratiques innovantes}

Le large spectre des activités d'apprentissage à l'école peut se diviser en deux grandes orientations liées au développement de l'enfant, les «tâches scolaires» (telles que les savoirs associés au contenu d'une matière) et les «buts intellectuels» (tels que les dispositions mentales utilisées pour interpréter des expériences et résoudre des problèmes) (Helm et Katz, 2000). Conformément à la conception du programme à Singapour, la promotion des capacités scolaires des élèves a priorité sur l'optimalisation de leurs dispositions mentales. Le programme standard, les manuels scolaires et les examens communs sont utilisés pour planifier l'enseignement et l'apprentissage des élèves et privilégier certaines connaissances particulières et fondamentales, au détriment d'autres savoirs. Même si la promotion des visées intellectuelles des élèves est également prise en compte à Singapour, les concepteurs de programmes ont tendance à associer ces dispositions mentales à l'apprentissage des matières, tout particulièrement dans les matières scolaires faisant l'objet d'examen, comme les mathématiques, les sciences et les langues - par exemple l'anglais et la «langue maternelle» de l'élève. Les activités du programme visent à assurer la réussite de l'apprentissage scolaire des élèves tout en y incluant, en une sorte de plusvalue, l'optimalisation de leurs dispositions mentales. Si l'on en croit l'emploi du temps d'un jour de classe dans les établissements scolaires primaires et secondaires généraux, l'apprentissage des matières régit la façon dont le temps et les activités sont organisés à l'école. Les résultats associés à l'apprentissage des élèves (qui seront expliqués plus en détail plus loin) sont évalués essentiellement au moyen d'examens sur feuille portant sur les savoirs liés à la matière en question.

De nouvelles politiques éducatives ont encouragé la mise en place de nouvelles initiatives d'enseignement et d'apprentissage pour soutenir les résultats scolaires de tous les élèves, au moyen d'un large spectre d'activités d'apprentissage. Par exemple, l'encouragement du gouvernement aux enseignants pour promouvoir les dispositions mentales des élèves (c'est-à-dire "enseigner moins, apprendre plus») entraîne une révision du programme incluant l'enseignement de la pensée critique, des compétences en technologies de l'information, et l'éducation civique (Koh, 2004). Cette initiative correspond à l'importance soulignée par Helm et Katz (2000) de développer les finalités intellectuelles des élèves plutôt que de se concentrer exclusivement sur les tâches scolaires. Par là-même, le défi lancé à la communauté éducative de Singapour est d'enseigner des contenus scolaires dans le but de préparer les élèves à des examens qui privilégient des savoirs spécifiques, tout en valorisant les divers talents et les capacités variées des élèves. En conséquence, cette prise de conscience et ces encouragements accrus pour mettre en ouvre des pratiques innovantes en matière d'enseignement et d'apprentissage ont pris toute leur 
importance dans les écoles de Singapour, alors que les enseignants luttent pour conjuguer tâches scolaires et visées intellectuelles, afin d'ouvrir de plus larges avenues menant à la réussite scolaire des élèves (Tan et Ng, 2005).

\section{De la transmission à l'apprentissage "transactionnel»}

Actuellement, Singapour affine et redéfinit la signification même des notions de savoir et d'apprentissage. Par là même, le modèle de Miller (1996) est utile pour rendre manifeste ce qui compte véritablement en termes de savoirs et pour déterminer comment ces savoirs sont valorisés par la synergie des contributions parmi les élèves, les enseignants et les décideurs de Singapour. Miller a indiqué qu'il existe trois formes de programmes pour donner forme et structure aux savoirs et aux apprentissages. Selon lui, les programmes «transformatifs» encouragent et guident l'apprentissage intégré et comprennent un large éventail de relations entre pensée et apprentissage - entre soi-même et l'univers, entre domaines de connaissance. Les programmes "transmissifs», d'autre part, se concentrent sur la précision et la validité des savoirs, alors que les programmes «transactionnels» s'attachent aux procédures d'apprentissage.

Autrefois, les connaissances que les élèves étaient censés posséder étaient acquises essentiellement grâce à une approche transmissive, par fragments de différentes matières, sous la houlette d'enseignants spécialistes de ces matières, fragments tirés principalement de manuels scolaires. Comme il est logique avec cette forme d'évaluation, on s'attend à ce que les élèves apportent la preuve de leur capacité à comprendre avec précision les savoirs associés à ces matières isolées. Comme nous en discuterons plus loin, l'accent mis sur les examens déterminant des filières au quatrième niveau de l'école primaire ainsi que le Primary School Leaving Examination (PSLE) ${ }^{2}$ poussent les élèves et leur famille à penser qu'ils doivent avoir les meilleurs résultats afin d'empêcher tout échec scolaire.

Le ministère de l'éducation et les programmes de formation des enseignants incitent actuellement les enseignants à valoriser les procédures d'apprentissage et à répondre aux divers besoins des élèves de manière à centrer la salle de classe sur l'enfant. Cette argumentation est alimentée par l'importance du processus d'apprentissage, ce qui correspond à une approche transactionnelle des programmes. Elle reconnaît la nécessité de redéfinir le métier d'enseignant, de dépasser la culture de résultats pour parvenir à un système éducatif orienté vers les processus. La notion, qui ne cesse d'évoluer, de ce que les enseignants entendent par apprentissage est déplacée vers les valeurs fondamentales d'une conception transactionnelle des programmes, conduisant les enseignants à

2. Examen de fin d'études primaires. 
adhérer à des valeurs libéralistes et à mettre en pratique les théories portant sur le développement de l'enfant. Par là même, ce que l'on entend par savoirs n'est guère compatible avec le fait que les élèves soient évalués et sanctionnés durant leur apprentissage (ce que font les examens standards) et que les enseignants doivent rendre compte de leur pratique pédagogique (qui est centrée sur l'élève et implique encore le besoin de l'aider à passer ses examens). Ce hiatus oblige les enseignants à faire le grand écart dans leur pratique pédagogique.

\section{L’apprentissage "transformatif "}

Selon Miller (1996), un programme holistique devrait associer les domaines de connaissance et les relations entre les individus et le monde dans lequel ils vivent, de manière à transformer ces connaissances et à les appliquer aux différents contextes où elles sont pertinentes. Ce que Miller vise, dans cette déclaration, s'applique aux récents efforts du gouvernement pour faire du pays une ville mondiale. Le gouvernement œuvre pour que Singapour soit considéré à l'échelle internationale comme un pôle économique et scientifique de premier plan. Il se rend pleinement compte qu'il est nécessaire que les habitants trouvent leur place dans les remous engendrés par un contexte mondial dont les mutations touchent croyances et cultures, si Singapour souhaite jouer un rôle important sur la scène mondiale (Koh, 2004; Sharpe et Gopinathan, 2002). Les décideurs locaux ont également adapté de nombreuses idées occidentales au contexte éducatif de Singapour. Ces efforts tendent à élargir les points de vue des gens afin de les connecter au monde, comme le proclame le slogan des festivités du National Day en 2006, "Connecting our homes, connecting global. ", " connecter nos foyers, se connecter au monde». Cependant, cette vision des fondamentaux en matière d'apprentissage comporte certaines béances en ce qui concerne les rôles joués par les élèves, les enseignants et les décideurs.

\section{L'ÉVALUATION À SingapouR}

\section{Les pratiques actuelles}

À Singapour, les élèves passent des examens nationaux à la fin de la sixième année du primaire, des quatrième et cinquième années du secondaire et de la seconde année du cycle pré-universitaire. L'examen de fin d'études primaires (Primary School Leaving Examination, ou PSLE) sert à évaluer les compétences scolaires des élèves pour leur faire suivre un cursus secondaire qui corresponde à leurs connaissances et leurs aptitudes scolaires. Dans le secondaire, les élèves qui suivent les cursus «Spécial» et «Express» passent le General Certificate of Education, "Normal» Level ou GCE N-Level à la fin de la quatrième année. Ceux qui réussissent bien peuvent alors poursuivre en 
cinquième année et présenter le O-Level (niveau ordinaire) du General Certificate of Education (GCE O-Level) Les élèves en fin d'études secondaires qui se destinent à l'université peuvent choisir l'une des deux solutions suivantes: suivre un cursus pré-universitaire de deux ans dans un établissement préparatoire, ou un cursus en trois ans dans un institut pré-universitaire centralisé. Ces deux filières conduisent à l'Advanced Level (niveau avancé) du General Certificate of Education, le GCE A-Level. Les élèves qui sont plus particulièrement intéressés par les études techniques ou commerciales peuvent rejoindre les instituts polytechniques pour suivre un cursus qualifiant, ou l'Institut d'enseignement technique (Institute of Technical Studies, ou ITE) afin de suivre des cours de formation technique. Enfin, ceux qui réussissent bien dans les instituts polytechniques et en ITE peuvent également suivre au choix un cursus universitaire ou qualifiant.

Les structures d'évaluation actuelles exigent des élèves singapouriens qu'ils étudient pour se préparer à des examens ordinaires, particulièrement à des tests écrits traditionnels, mettant l'accent sur des connaissances et des capacités fondamentales et standardisés en mathématiques, en anglais, en sciences et dans la «langue maternelle» de l'élève. Tous ces examens se passent en temps limité, se déroulent dans des conditions standardisées et sont presque tous du type «Prenez une feuille.»

Comme on peut l'observer sur le terrain, l'évaluation est utilisée par le gouvernement comme outil pour sélectionner les élites, et les élèves subissent de nombreux examens. Les enseignants et les élèves disposent d'une définition claire et précise de la norme des performances correspondant à chaque niveau d'enseignement dans le cadre du programme national. Le gouvernement a décidé que l'éducation primaire servait à préparer les Singapouriens aux connaissances et aux aptitudes fondamentales nécessaires au bon citoyen. L'éducation secondaire et tertiaire repose sur la croissance économique prévue et sur les besoins en main-d'œuvre (Gregory et Clarke, 2003). Cependant, il est important de remarquer que, "alors que le système éducatif de Singapour répond d'ores et déjà aux normes internationales en mathématiques, en informatique et dans les autres sciences, les experts ont signalé les performances relativement médiocres réalisées en termes de développement des aptitudes à la direction d'entreprise et de ressources humaines, ainsi qu'en termes de capacités à favoriser l'émergence de talents d'encadrement créatifs et dynamiques (Johnson, 2003, cité dans Tan, 2005, p. 12)».

\section{La nécessité de changer de modes d'évaluation}

Dans une certaine mesure, cette nouvelle politique éducative s'est efforcée de dépasser l'accent mis sur les examens, afin de gagner en souplesse et en diversité au sein du paysage éducatif singapourien. 
L'ex-premier ministre Goh Chok Tong (1997) a remarqué que les vieilles recettes à base d'apprentissage par cœur n'étaient guère susceptibles d'aider les jeunes Singapouriens à affronter de nouveaux contextes et de nouveaux problèmes. De plus, le rapport du très influent Comité économique du ministère du commerce et de l'industrie (1986) a fait apparaître que Singapour doit revoir et adapter l'enseignement dispensé à sa main-d'œuvre et produire davantage d'élèves et d'étudiants autonomes, créatifs et capables de s'adapter. Alors que le par cour et la hiérarchie, présents dans le confucianisme et les sociétés traditionnelles asiatiques, ont contribué à la stabilité de Singapour (Low, 2002), cette approche semble désormais inadaptée. La créativité et l'innovation sont valorisées parce qu'elles produisent des connaissances qui sont moins «incertaines » et moins difficiles à structurer, à conceptualiser et à mesurer. Leur acquisition va généralement de pair avec la prise de risques, un processus qui n'est guère encouragé par les approches éducatives traditionnelles.

Koh (2004) a fait valoir que les changements pédagogiques de Singapour dans les dix dernières années ont pour origine des impératifs planétaires. Le premier ministre Lee Hsien Loong (2004) a de fait admis cela lorsqu'il a proclamé que Singapour devait continuer à restructurer et moderniser son système éducatif pour tenir compte de la concurrence féroce engendrée par cette tendance générale à la mondialisation. Par là même, les écoles de Singapour se repositionnent pour répondre à des exigences à la fois différentes et nouvelles. Le gouvernement adhère à cette interconnexion entre les politiques économique et éducative. Les piliers de l'apprentissage et des connaissances des élèves ne sauraient continuer d'être édifiés de façon linéaire à partir de l'interprétation par le gouvernement des facteurs de réussite des élèves et de leur contribution à la croissance économique.

Singapour a besoin de citoyens capables d'une pensée plus créative et plus autonome pour répondre aux changements économiques mondiaux. Cependant, Sharpe et Gopinathan (2002) se sont posé la question de savoir si le système éducatif pouvait être restructuré pour produire de tels élèves tout en conservant les normes scolaires élevées exigées par le gouvernement. Dans la mesure où le système éducatif singapourien est extrêmement structuré et tributaire d'une culture des examens et des résultats, il est difficile d'enseigner et d'apprendre à penser dans un environnement aussi discursif. Koh (2004) a expliqué que la pensée critique a ses limitations pédagogiques et ne saurait totalement s'épanouir sous une pression gouvernementale normalisante. Parce qu'une croissance économique et une richesse minimales sont impératives en raison de la petite taille du pays et de sa limite démographique optimale de 5,5 millions d'habitants, le gouvernement singapourien cherche à trouver un juste équilibre entre les impératifs d'une mondialisation indispensable et sa propre identité sociale, politique et culturelle (Chun, 1996; Low, 2002). Il a besoin de temps pour passer à la vitesse supérieure, quitter un monde ancien, 
engendrant des générations de suiveurs, pour un monde nouveau, capable de produire des innovateurs qui ne craignent pas de prendre des risques, voire d'échouer. La discussion qui suit attire l'attention du lecteur sur une sélection d'exemples d'évolutions pédagogiques à Singapour.

\section{Le changement des modes d'évaluation}

La restructuration du système éducatif singapourien est en cours depuis le milieu des années quatre-vingts. Elle s'accélère, grâce à l'émergence de la mondialisation au cours des dernières années. Autrefois, le gouvernement se concentrait davantage sur la réussite des élèves aux examens dans le but de les sélectionner. Aujourd'hui, les résultats aux examens sont progressivement remplacés par des objectifs de stage et d'intervention. Le système éducatif singapourien s'assouplit progressivement pour devenir plus réceptif au changement grâce à des modes d'évaluation diversifiés.

L'évaluation fait partie intégrante du processus d'enseignement et d'apprentissage à l'école. Toutefois, le lancement de la vision dite «des écoles qui pensent, une nation qui apprend» a largement contribué à promouvoir révisions et changements en matière de pédagogie. Des procédures d'évaluation alternatives ont été ajoutées aux procédures existantes, complétant celles-ci. Les évaluations multiples évoluent progressivement.

Le programme de certaines matières a été réduit jusqu'à $20 \%$ pour dégager du temps afin de mettre en place des projets interdisciplinaires et des activités extrascolaires. Les établissements ont également été équipés d'ordinateurs et de didacticiels ; ils disposent d'une assistance technique. Les élèves passent jusqu'à $30 \%$ de leur temps scolaire à utiliser des ordinateurs (Sharpe et Gopinathan, 2002). De plus, la modification du contenu des enseignements et la réduction des programmes ont dégagé davantage d'espace et de temps pour mettre en place des activités créatives.

Cependant, dans la mesure où les établissements scolaires ont tendance à enseigner en fonction des évaluations, les programmes traditionnels continuent à régir la façon dont les élèves apprennent. À cause de résultats inattendus, la dissémination des meilleures pratiques pédagogiques par le «School Excellence Model» (modèle d'excellence scolaire) est également remise en cause. Les établissements ont tendance à reproduire les programmes «qui marchent ", sans guère se soucier de la spécificité de leurs propres élèves, souvent différents à maints égards. De plus, la coopération mutuelle et bénéfique entre établissements est freinée par la concurrence pernicieuse qu'ils pratiquent pour attirer les meilleurs enseignants et les meilleurs élèves. De surcroît, les parents ne sont pas toujours libres de leurs choix dans ce qu'ils édictent et dans ce à quoi ils consacrent leur temps et leur énergie, à cause de leur propre expérience, vécue au sein d'un système éducatif qui assure la compétitivité du pays dans une économie mondialisée. 


\section{PAR-DELÀ LES FRONTIÈRES DE L’ÉCOLE}

Villaverde (1999, p. 131) embrasse de nombreuses définitions lorsqu'il définit un programme comme "tout ce qui survient et qui est appris dans l'école, la salle de classe, à la maison ou dans la rue, de manière individuelle ou collective, ainsi que dans la société en général». Cependant, la notion de programme, à Singapour, semble faire référence à des manuels scolaires portant sur des matières uniques et des fiches de travail émanant de l'enseignant, à des expériences pédagogiques formelles menées au sein de la classe et à des activités extrascolaires conduites par le personnel d'encadrement scolaire. De manière spécifique, Zongyi et Gopinathan (2005, p. 32) affirment qu' «à Singapour, les enseignants tendent de façon conventionnelle à considérer le savoir comme un corpus de faits prouvés ou comme une information factuelle contenue dans des manuels scolaires approuvés par le gouvernement, à l'aune desquels les élèves sont testés durant les examens ».

Autrefois, on demandait aux parents d'encourager le développement de la réussite scolaire de leur(s) enfants(s) dans des disciplines uniques. Les nouvelles réformes pédagogiques présupposent que les parents encourageront à l'avenir le développement de toute une variété de talents, d'intérêts et de capacités. Cependant, le problème à Singapour est que la majorité des parents est à ce jour loin d'avoir renoncé à la prééminence de la préparation de leurs enfants aux examens. Comme le souligne Tan (2005, p. 7) «l'état d'esprit qui prévaut est que la réussite scolaire demeure primordiale». En fait, Tan apporte la preuve que le nombre d'établissements de soutien scolaire déclarés a augmenté de $86 \%$ au cours des cinq dernières années. Cela ne manque pas de soulever des questions, en cette période où le ministère de l'éducation s'est fait l'avocat d'initiatives plus innovantes et plus créatives dans les domaines de l'enseignement et de l'apprentissage et en a encouragé la mise en œuvre. Khong (2004, p. 7) a examiné le rôle des parents, et tout spécialement des mères, dans le système éducatif primaire singapourien, et la façon dont ils gèrent la scolarité de leurs enfants. Il nous offre les réflexions suivantes : "parce qu'à Singapour l'éducation est un sujet dont les enjeux sont considérables, la réussite scolaire demeurant le moyen le plus efficace pour se promouvoir socialement et économiquement, les parents prennent souvent l'initiative de pousser leurs enfants à lutter pour obtenir cette chaîne ininterrompue de $A^{3}$, tout spécialement lors d'examens cruciaux, comme le Primary School Leaving Examination (PSLE) ou les examens de type GCE-A Level ou O-Level».

Khong évoque les sommes élevées dépensées en cours particuliers de soutien à Singapour, parce que de nombreux foyers à double revenu disposent

3. La meilleure note possible, sur une échelle de $A$ (excellent) à $F$ (de «fail», échouer). La moyenne correspond à C et la note En'existe pas. 
de peu de temps et n'ont ni les compétences ni l'énergie nécessaires pour aider leurs enfants dans leur apprentissage scolaire. Tan (2005, p. 8) soutient également l'idée qu'un nombre non négligeable de parents d'enfants d'âge scolaire reconnaissent l'importance des acquis scolaires pour réussir dans la société singapourienne. "Il n'est pas inhabituel qu'un élève ordinaire d'école primaire suive des cours et des heures d'enseignement renforcé à l'école et passe trois heures par jour à remplir des cahiers d'exercices d'évaluation, plus deux autres heures de cours de soutien... L'état d'esprit dominant parmi les parents est que leurs enfants doivent avant toute chose réussir dans le domaine scolaire, et non dans les domaines périscolaires tels que les disciplines artistiques».

Les parents sont informés des résultats et du comportement de leur(s) enfant(s) à l'école et sont encouragés à travailler de conserve avec les enseignants pour préparer leur(s) enfants(s) au PSLE à l'issue du sixième niveau du primaire, afin de leur assurer une place dans un «bon» lycée "coté». De tels systèmes fondés sur la méritocratie ont contribué à favoriser un système éducatif hautement sélectif, poussant les parents à s'inquiéter sans cesse de la réussite scolaire de leurs enfants (Sharpe, 2000).

Les savoirs, les aptitudes, les attitudes et les capacités appris à l'école sont étroitement reliés aux apprentissages effectués hors de l'école. Le programme scolaire tel qu'il est défini influence l'apprentissage de nombreux élèves et étudiants singapouriens hors de l'école. Les interactions et les conversations, les événements et les expériences ainsi que les apprentissages et les savoirs des élèves durant leur vie extrascolaire se voient accorder une bien moindre importance, une bien moindre reconnaissance et constituent un terreau infiniment moins riche, une fois transposés dans l'espace des établissements primaires et secondaires.

\section{LA REMISE EN CAUSE DES PRATIQUeS ACTUELLES}

En tant qu'expatriés encadrant et supervisant les enseignants en formation et en service, et que chercheurs et consultants spécialistes des pratiques d'enseignement et d'apprentissage, nous accordons au système éducatif tout le respect qui lui est dû et ne manquons pas d'apprécier à sa juste valeur son rôle historique ainsi que les changements actuellement mis en œuvre. Comme étrangers, nous sommes également conscients et soucieux du fait que notre compte rendu peut éventuellement comporter des généralisations et se voir déformé par une vision taïwanaise, nord-américaine et australienne - nos pays respectifs d'origine ou de formation. Néanmoins, «tout programme suppose toujours définition, redéfinition et négociation» (Teitelbaum, 2004, p. 127) et, comme enquêteurs travaillant sur les programmes et sur les mises au point pédagogiques, chacun dans notre rôle, nous ressentons la nécessité de suggérer les commentaires et les questions suivants pour susciter un débat contradictoire permanent sur les savoirs, l'enseignement et les apprentissages à Singapour. 


\section{Réflexions sur les programmes et l'évaluation}

Alors que le système éducatif a connu des changements - les plus récents étant ceux apportés à l'architecture et à la structure des regroupements d'établissements, à l'amélioration des espaces d'enseignement, à la réduction de la taille des classes, et à la formation des maîtres (Ho, 2006) - les initiatives introduisant de la souplesse dans la gestion et de l'aide pour tous les élèves sont contrariées par le maintien d'examen et de filières.

Le ministère de l'éducation souligne l'importance de respecter toutes les formes de talents (Tharman, 2004 et 2006) et de satisfaire toutes les capacités des élèves (Teo, 2001). En fait, le premier ministre Lee (ministère de l'éducation, 2006) a fait état lors du Teachers Day Rally (réunion tenue lors de la Journée des enseignants) de l'importance de parvenir à constituer «de nombreux pôles d'excellence pour les enfants présentant des capacités, des aptitudes et des intérêts différents ». Toutefois, les universitaires reconnaissent qu'un programme fondé sur les capacités ne reflète guère la réalité et que l'état d'esprit qui prévaut chez de nombreux enseignants et de nombreux parents demeure inchangé - à savoir que la réussite scolaire, en particulier en mathématiques et en sciences, demeure primordiale (Tan, 2005). Comment faire pour que d'autres formes de connaissances et d'apprentissages, telles que les humanités (littérature, histoire et art, par exemple) soient assimilées et considérées au même titre que les sciences, les mathématiques et la technologie dans les écoles singapouriennes? Voulons-nous que nos enfants fassent partie d'une société fondée sur des théories économiques, ou sur le bien-être de l'homme? Comme Apple (2006, p. 23) le montre, "pour favoriser une économie de marché, nous devons encourager chacun à penser en tant qu'individu qui n'agit que pour maximaliser ses propres intérêts ». Apple nous pousse à nous demander si nous voulons d'un système qui fonctionne en considérant qu'il est parfaitement normal d'avoir des gagnants et des perdants. La culture devrait-elle présider à l'éducation, ou l'éducation à la culture? Dans le cadre d'une vision à plus long terme du pays et de ses habitants, la politique économique devrait-elle être définie avant de fixer la politique éducative, ou l'inverse? Quel rôle peut jouer l'éducation dans le développement de l'identité de l'élève au moyen des cultures et des normes traditionnelles, tout en facilitant sa pensée créatrice - qui peut très bien entrer en conflit avec ces mêmes valeurs traditionnelles?

Enfin, un rapport récent du World Bank Institute (Kaufmann, Kraay et Mastruzzi, 2006) a montré que Singapour devait être davantage responsable et transparente en matière de choix politiques. Toute politique implique de traduire les demandes et les propositions en matière d'initiative pédagogique en changements tangibles dans les pratiques pédagogiques sur le terrain (dans les relations entre élèves et enseignants comme en termes de savoirs et d'apprentissages). Cependant, comme dans le cadre ancien des traditions d'examen, la 
majorité des établissements scolaires de Singapour continue de se concentrer sur ce qui est quantifiable. Afin de modifier les pratiques d'enseignement et d'apprentissage, les fondements de ces pratiques doivent eux aussi être modifiés, à la fois pour les élèves et pour les écoles (Teo, 1999). De quelle façon doit-on utiliser les évaluations multiples pour brosser un tableau plus complet, une description plus détaillée, des capacités et des aptitudes d'un élève? Comment faire pour que l'apprentissage et les résultats d'un élève forment un tout cohérent et continu à travers les différents niveaux du système éducatif singapourien? Les tests traditionnels du type «Prenez une feuille» ne sont plus guère adaptés pour évaluer certaines des aptitudes et des savoirs nécessaires dans une économie mondialisée. De plus, comment les écoles singapouriennes peuventelles reconnaître, apprécier, et intégrer les savoirs et les apprentissages des élèves dans un cadre extrascolaire, avant de prendre appui sur ces derniers? Peut-on redessiner les frontières séparant l'école de la maison? Comment peut-on aider les enseignants à mettre en œuvre les nouvelles directives du programme, dans le but d'améliorer enseignement et apprentissage et de rendre le travail des élèves plus fructueux?

\section{BIBLIOGRAPHIE}

APPLE M.W. (2006) : Understanding and interrupting neoliberalism and neoconservatism in education, Pedagogies: An International Journal, 1 (1), 21-26.

BOSHIER R. (1994): Education and docility: The dilemmas of Singapore and the next lap. In: N. P. Stromquist (ed.) Education in urban areas: Cross-national dimensions (pp. 131-150). Westport, Conn. : Praeger.

CHUA S.K.C. (2004, November): The convergence and divergence effects of globalization on Singapore's education system. Paper presented at the 2004 annual meeting of the Australian Association for Research in Education, Melbourne, Australia.

CHUN A. (1996): Discourse of identity in the changing spaces of public culture in Taiwan, Hong Kong and Singapore. Theory, Culture and Society, 13, 51-75.

GOH C.T. (1997) : Shaping our future: "Thinking Schools" and a "Learning Nation". Speeches, 21, 12-20. Singapore : Ministry of Education.

GREGORY K. \& CLARKE M. (2003): High-Stakes assessment in England and Singapore. Theory into Practice, 42, 66-74.

HELM J. H. \& KATZ L. (2001): Young investigator: The project approach in the early years. New York : Teachers College Press.

KAUFMANN D., KRAAY A. \& MASTRUZZI M. (2006): Governance Matters V: Governance indicators for 1996-2005. World Bank Policy Research.

KLEIN P. (1996): Cross-cultural experiences with a mediational approach. New York \& London : Garland Publishing, Inc ISBN : 0-8153-1244-X.

KOH A. (2004) : Singapore education in "New Times": Global/local imperatives. Discourse: Studies in the Cultural Politics of Education, 25, 335-349. 
LOW L. (2002): Globalization and the political economy of Singapore's policy on foreign talent and high skills. Journal of Education and Work, 15, 409-425.

MILLER J. (1996): Holistic curriculum. Toronto : Oise Press.

The Ministry of Education, Singapore (2006): Speech by Prime Minister Lee Hsien Loong at the Teachers' Day Rally 2006. Retrieved September 08, 2006, from http://www.moe. gov.sg/speeches/2006/sp20060831.htm

The Ministry of Education, Singapore (2005): Mission and vision statement. Retrieved September 08, 2006, fromhttp://www.moe.gov.sg/corporate/mission_statement.htm

MUKHOPADHAYA P. (2003): Trends in income disparity and equality enhancing (?) education policies in the development stages of Singapore. International Journal of Educational Development, 23, 37-56.

SHARPE L. \& GOPINATHAN S. (2002): After effectiveness: New directions in the Singapore school system? Journal of Education Policy, 17, 151-166.

TAN C. (2005): The potential of Singapore's ability driven education to prepare students for a knowledge economy. International Education Journal, 6, 446-453.

TAN C. (2005): Driven by pragmatism : Issues and challenges in an ability-driven education. In: J. Tan and P.T. Ng (Ed.). Shaping Singapore's Future: Thinking Schools, Learning Nation (pp. 5-21). Singapore : Prentice Hall.

TAN J. \& Ng P.T. (2005): Shaping Singapore's Future: Thinking Schools, Learning Nation. Singapore : Prentice Hall.

TEO C. H. (2001): "Making an ability driven education happen". Ministry of Education FY2001 Committee of Supply Debate. Minister's First Reply on Schools. Retrieved September 08, 2006, fromhttp://www.moe.gov.sg/speeches/2001/sp15032001.htm

TEO C. H. (1999): Ministerial Statement by the Minister for Education, Teo Chee Hean at the Committee of Supply Debate, FY 1999 in Parliament, 17 March 1999.

TEITELBAUM K. (2004): Curriculum theorizing. In: J.L. Kincheloe and D. Weil (Eds.), Critical Thinking and Learning: An Encyclopaedia for Parents and Teachers (pp. 127-131). Westport, CT : Greenwood Press.

THARMAN S. (2006): Speech at the work plan seminar 2006. Retrieved September 30, 2006, from http://www.moe.gov.sg/speeches/2006/sp20060928.htm

THARMAN S. (2004): Speech at the work plan seminar 2004. Retrieved September 08, 2006, from http://www.moe.gov.sg/speeches/2004/sp20040929.htm

TYLER R. W. (1949): Basic principles of curriculum and instruction. Chicago : University of Chicago Press.

VILLAVERDE L.E., KINCHELOE J.L. \& STEINBERG S. (1999): Rethinking intelligence: Confronting psychological assumptions about teaching and learning. New York: Routledge.

ZONGYI D. and GOPINATHAN S. (2005): The information technology masterplan. In: J. Tan and P.T. Ng (Ed.). Shaping Singapore's Future: Thinking Schools, Learning Nation (pp. 22-40). Singapore : Prentice Hall. 